\title{
Functional outcome of an arthroscopic anatomical single bundle anterior cruciate ligament reconstruction using semi-tendinosus graft with fixation using endo-button on femoral side and suture disc on tibial side: a prospective clinical study
}

\author{
Vinod Jagtap $^{1}$, Namdev Gorgile ${ }^{1}$, Yash Shah ${ }^{2} *$, Vikas Rokade ${ }^{1}$, Girish Bartakke ${ }^{1}$
}

\author{
${ }^{1}$ Department of Orthopaedics, GMC and civil Hospital, Miraj, Maharashtra, India \\ ${ }^{2}$ MIMER Medical College, Talegaon, Pune, Maharashtra, India
}

Received: 12 August 2017

Revised: 30 September 2017

Accepted: 02 October 2017

\section{*Correspondence:}

Dr. Yash Shah,

E-mail: dr.yashshah@gmail.com

Copyright: () the author(s), publisher and licensee Medip Academy. This is an open-access article distributed under the terms of the Creative Commons Attribution Non-Commercial License, which permits unrestricted non-commercial use, distribution, and reproduction in any medium, provided the original work is properly cited.

\begin{abstract}
Background: Injury to the anterior cruciate ligament (ACL) is one of the most frequent injuries of the knee during different sports activities. Arthroscopic surgical reconstruction is the current standard of care for treatment of ACL injuries in young and active patients. The widespread adoption of ACL reconstruction over primary repair was based on early perception of the limited healing capacity of the ACL. Hamstring tendon is most favoured graft for ACL reconstruction. We wanted to study the outcomes particular method of fixation for fixing the hamstring tendon.

Methods: In this study 30 patient with ACL injury were treated arthroscopically for the fixation of graft in bone tunnel we use endobutton for femur and suture disc for tibia as suspensor implant and prospective assessment of functional outcome using Tegner Lysholm knee scoring system.

Results: The preoperative activity level could be maintained in $70 \%$ of the patients. The Lysholm score showed very good and good results in $80 \%$. Functional and stability results in about $70 \%$ of the patients.

Conclusions: This surgical technique can be recommended for the active patient with ACL deficiency. The functional outcome of anterior cruciate ligament reconstruction with quadrupled semitendinosus tendon autograft using Endobutton and suture disc is good. This method of fixation will help the graft to facilitate graft tunnel healing and also maintain its strength until there is a good graft to bone healing occurs completely.
\end{abstract}

Keywords: Cruciate, Ligaments, Endobutton, Suture disc

\section{INTRODUCTION}

Anterior cruciate ligament rupture is the most common knee ligament injury. The incidence of ACL tears has increased in the general population with the rise of participation in sports. The development of symptomatic knee instability after ACL injury ranges from $16 \%$ to almost $100 \%$. There have been significant technical advances during recent decades to treat ACL insufficiency and many studies have documented the successful results of contemporary arthroscopic ACL reconstruction. ${ }^{1,2}$ Bone-patellar tendon-bone (BPTB) or hamstring constructs are most commonly used. All autologous graft has donor site morbidity. Many studies have demonstrated that hamstring grafts have fewer problems with anterior knee pain, quadriceps muscle deficits, loss of extension compared with BPTB autografts. Low harvest morbidity and excellent biomechanical graft properties coupled with improved fixation of soft tissue grafts are all reasons for excellent 
clinical outcomes of ACL reconstruction using hamstring tendons. $^{3,4}$

Suspensory methods using Endobutton for femur and suture disc for tibia tunnel fixation and aperture methods of fixation have been described, with aperture fixation resulting in increased stiffness of the construction compared with the suspensory method. ${ }^{5,6}$

\section{METHODS}

Our study was designed to analyse the postoperative outcome of arthroscopic ACL reconstruction with quadrupled semitendinosus tendon autograft fixed in femoral tunnel using Endobutton and in the tibial side using suture disc.

The study was retrospective in nature and was conducted at GMC and Civil hospital MIRAJ between April 2013 to April 2016. In this study 30 patients who suffered from ACL injury were diagnosed and operated arthroscopically for anatomical single bundle anterior cruciate ligament (ACL) reconstruction using semitendinosus graft with fixation using Endobutton on femoral side and suture disc on tibial side.

\section{Exclusion criteria}

Age >45 years, previously operated knee, ligament reconstruction of the contralateral knee, associated lateral or medial ligament lesions, as well as observed chondral lesions that could modify the postoperative rehabilitation protocol (grade III or IV cartilaginous lesions).

\section{Pre-operative assessment}

The pre-operative assessment included detailed history and physical examination as Lachmann test, anterior drawer test and Mc Intosh Pivot shift test were used for testing ACL. The results were graded as 0 (negative), 1+, $2+$ and $3+$ positive. Other tests performed routinely included the varus and valgus stress tests, Mc Murray's test, posterior drawer test and the reverse pivot shift test. Radiographs included the standing AP view of both the knees and a lateral view of the affected. MRI of the involved knee was done in all the cases. In all acute cases of ACL injury, patient were treated with long knee brace for three weeks following which ACL reconstruction was planned once the patient regained full ROM with reduction of knee swelling.

\section{Operative technique}

\section{Arthroscopic technique}

After induction of anaesthesia, supine position with upper thigh tourniquet. Clinical tests performed under anaesthesia. Initial diagnostic arthroscopy performed via anteromedial and high anterolateral portal, after the confirmation of ACL tear arthroscopically, we preceded with semi-T graft harvest through oblique incision about $3 \mathrm{~cm}$ antero-medially on proximal tibia starting $3-4 \mathrm{~cm}$ distal to joint line and $3-4 \mathrm{~cm}$ medially to tibial tuberosity. Semitendinosus tendon is more horizontal \& flat and lies below gracilis which is round. It is pulled with curved clamp or a mixtard and freed end of graft whipstitched with size 5 Ethibond (Ethicon J\&J) suture, using closed tendon stripper semi-T graft harvested after releasing the vinculae. The quadrupled graft prepared over graft preparation board using size 5 Ethibond and the same used for pre-tensioning to reduce creep and relaxation. Usually the overall length of the tendon is $26 \mathrm{~cm}$ to 32 $\mathrm{cm}$. Once the graft quadrupled its size measured with the graft sizer, after the confirmation of graft size. Femoral tunnel preparation is done through anteromedial port with knee in maximum flexion (>120 degree). The guide pin advanced so that it exits the distal femoral cortex the femoral tunnel length to be reamed is measured with a depth gauge and then calculated according to the length of the graft material. Using the appropriate diameter reamer, the femoral tunnel is reamed based on graft size by keeping the minimum of $5 \mathrm{~mm}$ femoral cortex intact. Tibial guide placed taking ACL footprint, the inner edge of anterior horn of the lateral meniscus, the medial tibial spine, the PCL as reference points. Guide pin passed and confirmed its position arthroscopically in maximum extension to prevent impingement. Cannulated reamer over it is determined by the prepared graft diameter size. Initially No. 2 ethibond attached to eye of beath pin passed through anteromedial port to the femoral tunnel exiting lateral aspect of thigh. Using suture retriever through tibial tunnel pull the other end of ethibond out and secure to the pre-tensioned graft with endobutton attached and is pulled out of the femoral tunnel, the threads of the endobutton are pulled using the principle of flipping the endobutton and the femoral fixation is confirmed by togging of the endobutton. By keeping uniform tension on the grafts, the knee is taken through approximately 15 to 20 cycles of complete flexion and extension. This helps to align the grafts and reduce its creep and relaxation. The tibial side of the graft is fixed with a suture disc which is held over the tibial tunnel by passing the ethibond threads through the suture disc and tightening the knots around the disc. The joint is cleared off the debris by thorough lavage. Graft harvest site \& portals sutured in layers with no 2-0 vicryl and 2-0 ethilon/skin staples and thereafter dressing is done and compression bandage is applied and tourniquet deflated. Long knee extension brace applied.

\section{Rehabilitation}

Postoperative protocol for rehabilitation was used. A brace was given for a period of three week to protect the ligament. Range of motion of the knee and isometric muscle exercises were started the day after the operation and gradually progressed on the basis of closed kinetic chain exercises. Knee flexion of more than $90^{\circ}$ and walking with full weight bearing was allowed one month postoperatively. Indoor cycling and swimming were 
permitted after four weeks and running after 3 months. High demand sports activities were allowed after approximately 12 months.

\section{RESULTS}

This series included 30 patients; the minimum follow-up of the clinical assessment was 1 years with mean follow up of 30 month.

Highest number of patients fall in age group 21-25 yrs (30\%), next being 26-35 yrs (50\%). 35-45 yrs (20\%). Elder patients aged $45 \mathrm{yrs}$ and younger ones are $21 \mathrm{yrs}$.

Male patients account to $27(90 \%)$ of the total 30in study group and rest $3(10 \%)$ are females. Male preponderance may be related to their frequent involvement in outdoor work and strenuous activities. 18 out of 30 patients $(60 \%)$ got right knee involvement and 12 left knee ACL tear (40\%). Nature of injury in 12 patients $(40 \%)$ is due to sports (athlete, joggers, jump, physical trainers, kabaddi, cricket, players). RTA being the cause for ACL tears in $12(40 \%)$ patients and the rest $6(20 \%)$ sustained injuries due to other causes like slip and fall. All the patients were examined with Lachmann, anterior drawer and pivot shift tests in OPD and under anaesthesia prior to surgery. In OPD, $24(80 \%)$ patients turned positive Lachmann test 6 (20\%) patients Lachmann negative. Under anaesthesia all were Lachmann positive. In OPD 7 were anterior drawer positive, when the same done under anaesthesia 10 were positive. Pivot shift test was positive in $18(60 \%)$ patients in OPD but the same when done under anaesthesia was positive in 30 out of total 30 patients. Results of associated injuries on initial diagnostic arthroscopy shows ACL with MM tear in 15 patients (50\%), isolated ACL tear in 12 patients (40\%). ACL tear with MM and LM injury was noted in $3(10 \%)$ patient. Complications at the end of 6 months 2 patients complained of mild pain at the graft site. No superficial or deep infection seen. Lachmann grade I laxity seen in 21 patients and grade II in 6 patients at the end of final follow up but none complained of instability. 2 patients (who had preoperative restriction of terminal flexion of about 10 degrees) had restriction of the terminal 10-15 degree flexion at the postoperative 6 month follow-up and 2 patients had 5-10 degree restriction of extension, which improved with physiotherapy.

Postoperative X-rays were taken, and all the patients were examined 3, 6, 12 and 24 months after surgery as well as at the latest follow-up. Tunnel widening at the tibial side as well as femoral side was not seen. The clinical assessment was based on, Lysholm score. In our study, $21(70 \%)$ patients got normal postoperative recovery according to Lysholm score scoring at final follow-up, while 6(20\%) near normal and rest $3(10 \%)$ were abnormal related to knee stiffness and 7 patients got excellent results according to Lysholm score scoring while 6 patients showed good results, and the score was fair in 3 patient at the final follow up as shown in Table 1.

Table 1: Clinical assessment after 1 year follows up.

\begin{tabular}{|lllll|}
\hline No of patients & Range of movements & Lachmann laxity grade & Lysholm score & Results \\
\hline $\mathbf{2 1}$ & 140 & Grade I & $95-100$ & Excellent \\
\hline $\mathbf{6}$ & $120-140$ & Grade II & $84-94$ & Good \\
\hline $\mathbf{3}$ & 90 & Grade II & $<83$ & Fair \\
\hline
\end{tabular}
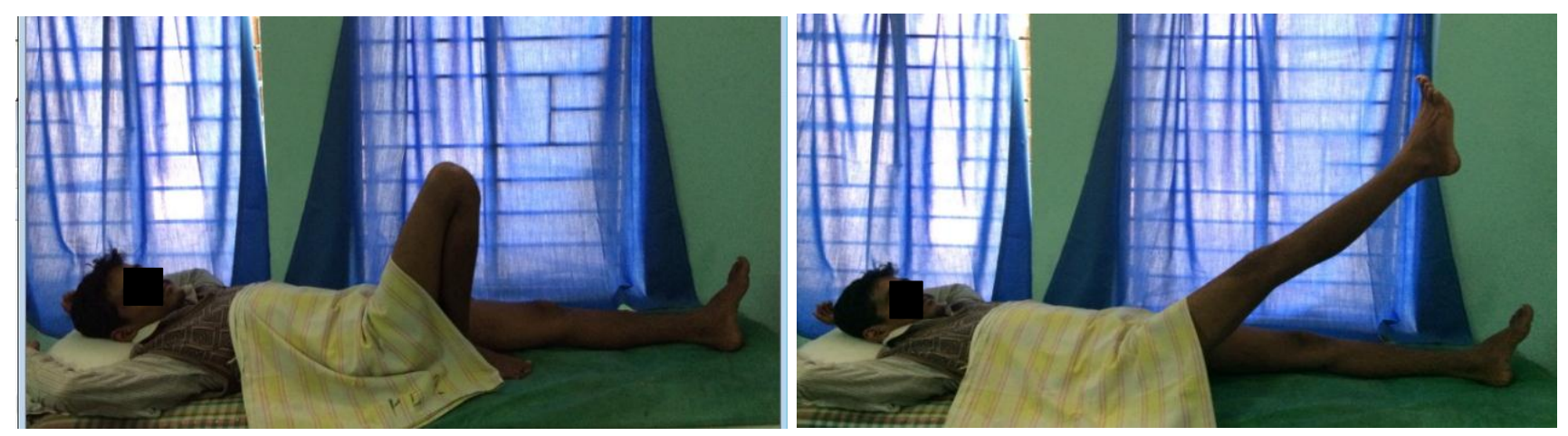

Figure 1: Range of movements.

\section{DISCUSSION}

Surgical reconstruction of the ACL has become the standard in the treatment of ACL injuries. The primary goal of ACL reconstruction is to restore the stability of the knee. Successful clinical outcomes following anterior cruciate ligament reconstruction with a hamstring graft has been reported by many authors. ${ }^{7,8}$

The choice of fixation in ACL reconstruction is still evolving and the current fixation device which has been widely used were the Endo button and the Bio composite 
interference screws which has helped to render an improved rehabilitation program post operatively. ${ }^{9}$ All patients in our study underwent ACL reconstruction with hamstring graft fixed with endobutton and suture disc for tibial tunnel. Cooley et al in their study concluded that ACL reconstruction using quadrupled fold semitendinosus tendon autograft provides excellent clinical outcome, patients maintain pre-injury activity without episodes of re-injury. ${ }^{10}$ Degeneration of the articular surfaces rarely occurs and the re-operation rates are minimal. About $85 \%$ of the patients according to Lysholm scoring fall into normal to near normal in their study as compared to more than $70 \%$ normal and $23 \%$ near normal in our study. Although endobutton is not a direct fixation device into the graft, there is a nylon material present between the graft and the button. This suspensory fixation has been associated with increased anterior joint laxity. ${ }^{8}$ In our study we found 7 patients had grade I laxity after 1 year grade II in 3patients at the end final follow up. Due to the indirect fixation of the graft there is a cyclic stretching of the graft under load which leads to the tunnel widening and inhibit the tendon-bone healing. ${ }^{10}$ There is an anteroposterior movement occurring inside the widened tunnel described as the "windscreen wiper" effect. Nebeking et al has described that there will be femoral tunnel enlargement when fixing the hamstring tendon with Endobutton in his study. ${ }^{11}$ In our study we had no tibial tunnel widening noted

The advantages of biodegradable interference screw is maintained as we have used titanium suture disc which is also MRI compatible and revision surgery is much easier when compared to metal screws. The disadvantage is breakage of the screw while inserting and the biocompatibility screw is concern which can be overcome with suture disc usage. ${ }^{12,13}$

In a study by Spicer et al areas of sensory change over the front of the knee were identifiable in $50 \%$ of the patients and of these $86 \%$ demonstrated sensory changes in the distribution of the infra-genicular branch of the saphenous nerve. ${ }^{14}$ In our study patients complained of numbness over the anterior aspect of leg around $10 \%$ of patients.

\section{CONCLUSION}

This study was conducted on 30 patients suffering from ACL deficiency in the age group of 19-45 years. Instability of knee in the form of giving way is main complaint evaluated by Lachmann test and confirmed by arthroscopy. The functional outcome of anterior cruciate ligament reconstruction with quadrupled semitendinosus tendon autograft using endobutton and suture disc will help the graft to facilitate graft tunnel healing and also maintain its strength until there is a good graft to bone healing occurs completely is excellent (70\%) with grade I laxity and grade II laxity in $30 \%$ and excellent functional outcome in $70 \%$ and good in $20 \%$ as per Lysholm score.

\section{ACKNOWLEDGEMENTS}

We give thanks to Government Medical College, Miraj and its entire staff.

\author{
Funding: No funding sources \\ Conflict of interest: None declared \\ Ethical approval: Not required
}

\section{REFERENCES}

1. Apostolopoulos A, Nakos A, Nikolopaulos D, Theophanopaulos F, Liarokapis S, et al. ACL reconstruction with hamstring tendon autografts. EEXOT. 2009;60(1):48-52.

2. Frank CB, Jackson DW. The science of reconstruction of the anterior cruciate ligament. J Bone Joint Surg Am. 1997;79(10):1556-76.

3. Chen L, Cooley V, Rosenberg T. ACL reconstruction with hamstring tendon Orthop Clin North Am. 2003;34(1):9-18.

4. Lawhorn KW, Howel SM. Principles for using hamstring tendons for anterior cruciate ligament reconstruction. Clin in Sports Med. 2007;26(4):56785.

5. Prodromos CC, Han YS, Keller BL, Bolyard RJ. Stability results of hamstring anterior cruciate ligament reconstruction at 2- to 8-year follow-up. Arthroscopy. 2005;21(2):138-46.

6. Ishibashi Y, Rudy TW, Livesay FA, Stone JD, Fu FH, Woo SL. The effect of anterior cruciate ligament graft fixation sites at the tibia on knee stability: Evaluation using a robotic testing system. Arthroscopy. 1997;13(2):177-82.

7. Aglietti P, Buzzi R, Zaccherotti G, De Biase P. Patellar tendon versus doubled semitendinosus and gracilis tendons for anterior cruciate ligament reconstruction. Am J Sports Med. 1994;22:211-7.

8. Aglietti P, Buzzi R, Menchetti PM, Giron F. Arthroscopically assisted semitendinosus and gracilis tendon graft in reconstruction for acute anterior cruciate ligament injuries in athletes. Am J Sports Med. 1996;24:726-31.

9. Freedman KB, D'Amato MJ, Nedeff DD, Kaz A, Bach BR Jr. Arthroscopic anterior cruciate ligament reconstruction. A meta-analysis comparing patellar tendon and hamstring tendon autografts. Am J Sports Med. 2003;31(1):2-11

10. Cooley V, Deffner K, Rosenberg T. Quadrupled semitendinosus anterior cruciate ligament reconstruction: 5-year results in patients without meniscus loss. Arthroscopy. 2001; 17:795-800.

11. Nebeking W, Becker R, Meckel M, Ropke M. Bone tunnel enlargement after anterior cruciate ligament reconstruction with semitendinosus tendon using Endobutton fixation on the femoral side. Arthroscopy. 1998;14(8):810-5.

12. Harvey A, Thomas NP, Amis AA. Fixation of the graft in reconstruction of the anterior cruciate 
ligament. J Bone Joint Surg Br. 2005;87(5):593603.

13. Clatworthy MG, Annear P, Bulow JU, Bartlett RJ. Tunnel widening in anterior cruciate ligament reconstruction: a prospective evaluation of hamstring and patellar tendon grafts. Knee Surg Sports Traumatol Arthrosc. 1999;7:138-45.

14. Spicer DDM, Blagg SE, Unwin AJ, Allum RL. Anterior knee symptoms after four-strand hamstring tendon anterior cruciate ligament reconstruction.
Knee Surg Sports Traumatol Arthrosc. 2000;8:2862.

Cite this article as: Jagtap V, Gorgile N, Shah Y, Rokade V, Bartakke G. Functional outcome of an arthroscopic anatomical single bundle anterior cruciate ligament reconstruction using semi-tendinosus graft with fixation using endo-button on femoral side and suture disc on tibial side: a prospective clinical study. Int J Res Orthop 2017;3:1175-9. 